\title{
Medical Image of the Week: PSG Sweat Artifact
}

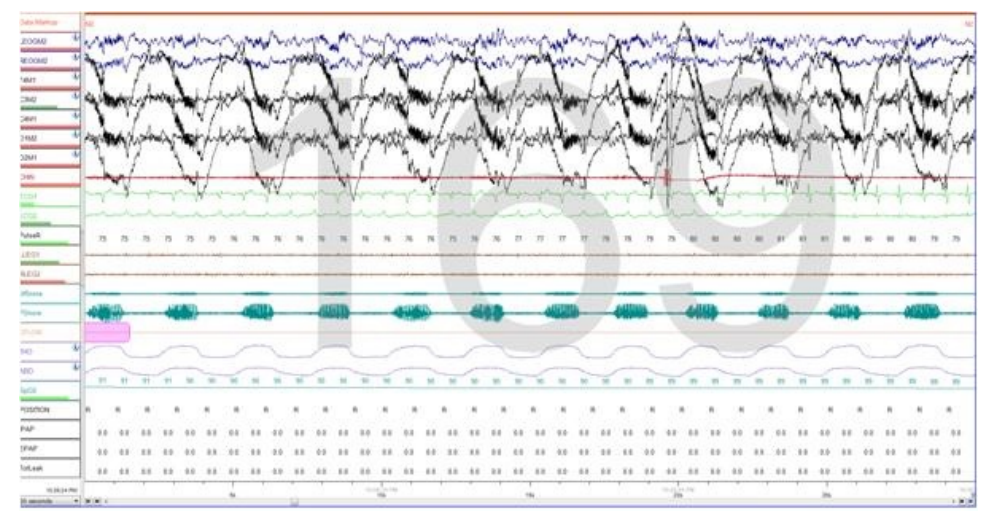

Figure 1. A 30 second epoch suggestive of sweat artifact and incidentally noted snore artifact on the M1 channels.

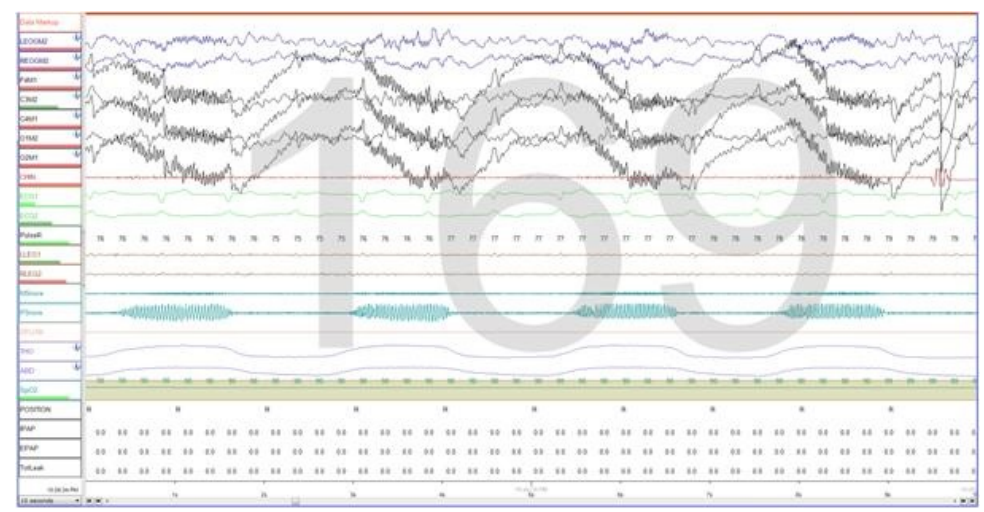

Figure 2: Sweat artifact as seen in a 10 second epoch.

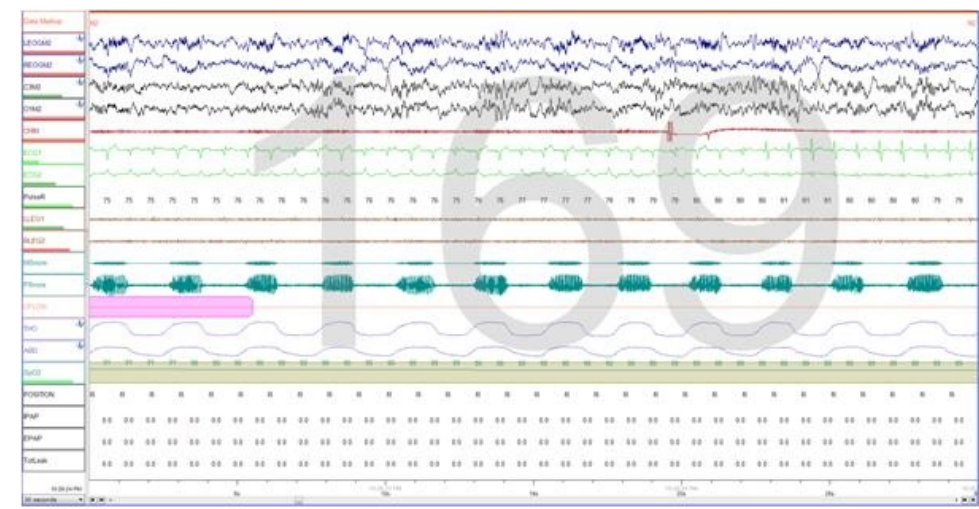

Figure 3. 30 second epoch after removal of the M1 channels. 
A 61-year-old man, with a past medical history significant for hypertension, COPD and morbid obesity with a body mass index (BMI) of 45.81 is referred for an overnight sleep study for suspicion of obstructive sleep apnea. Artifact was noted on the polysomnogram recording as shown above (Figures 1-3).

Sweat artifact is characterized by slow undulating movement of the baseline recording in the affected channels due to perspiration altering the potential of the involved electrodes (1). Sweat artifact may mimic delta waves and scored as non-rapid eye movement (NREM) stage 3 sleep. Lowering the room temperature, using a fan on the scalp or replacing the conductive paste on the electrodes may help eliminate the artifact.

Safal Shetty, $M D^{1}$ and John Roehrs, $M D^{2}$

${ }^{1}$ Banner University Medical Center Tucson, AZ

${ }^{2}$ Southern Arizona VA Health Care System

Tucson, AZ

\section{Reference}

1. Siddiqui F, Osuna E, Walters AS, Chokroverty S. Sweat artifact and respiratory artifact occurring simultaneously in polysomnogram. Sleep Med. 2006;7(2):197-9. [CrossRef] [PubMed] 